\title{
Cartas de control con variables convolucionadas
}

Control charts with convolutioned variables

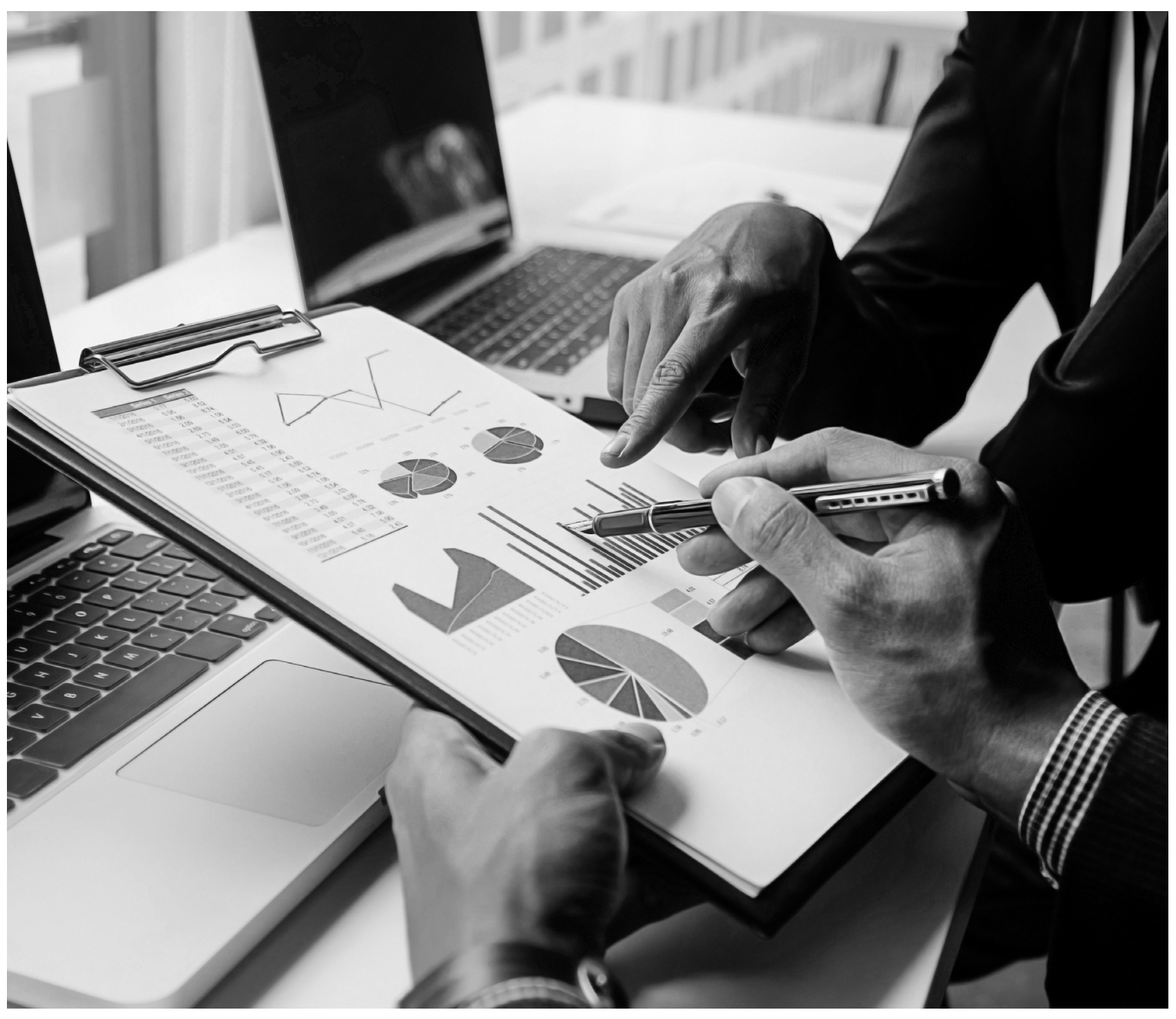




\title{
Cartas de control con variables convolucionadas ${ }^{1}$ Control charts with convolutioned variables
}

\section{Roberto José Herrera Acosta ${ }^{2}$, Paola Milena Rojas Manga ${ }^{3}$, Karla Patricia Jiménez Moreno ${ }^{4}$}

Artículo recibido en diciembre de 2017; artículo aceptado en septiembre de 2018.

Este artículo puede compartirse bajo la Licencia Creative Commons Atribución-NoComercial-Compartirlgual 4.0 Internacional y se referencia usando el siguiente formato: Herrera, R. J., Rojas, P. M. \& Jiménez, K. P. (2019). Cartas de control con variables convolucionadas.

I+D Revista de Investigaciones, 13 (1), 98-105. DOI: https://doi.org/10.33304/revinv.v13n1-2019008

\begin{abstract}
Resumen
El objetivo del presente documento es presentar, en el ámbito del control estadístico de calidad, una aplicación de las cartas de control Shewhart con más de una variable, utilizando la convolución como estadístico. Este trabajo selecciona un caso de estudio en el que se tienen dos variables: los diámetros de un resorte de compresión; y una nueva variable obtenida mediante convolución, a la cual se le hará monitoreo a través una carta de control univariada. Se comparan las cartas de control para datos individuales, en este caso tomando los diámetros del resorte por separado, y elaborando la carta de rango móvil, tomando como estadístico de control el valor generado por la convolución entre estas variables. Los resultados muestran que las cartas convolucionadas minimizan el impacto de una característica de calidad afectada por un factor ajeno a las condiciones naturales del proceso.
\end{abstract}

Palabras clave: carta de control, convolución, estadístico de control, monitoreo, señal de alarma.

\begin{abstract}
The objective of this document is to present, in the field of statistical quality control, an application of the Shewhart control charts, with more than one variable, using the convolution as a statistic. This paper selects a case study, in which there are two variables: the diameters of a comprehension spring; and a new variable is obtained by convolution, which will be monitoring through a univariate control chart. A comparison is made between the control charts, for individual data, in this case taking the diameters of the spring separately, and elaborating the mobile range chart, taking as control statistic the value generated by the convolution between these variables. The results show that charts convolved, minimize the impact that a quality feature affected by a factor other than the natural conditions of the process.
\end{abstract}

Keywords: control char, convolution, control statistics, monitoring, alarm signal.

1. Reporte de caso, tipo de enfoque cuantitativo, resultado del proyecto de investigación Nuevas Cartas de Control en curso, perteneciente al área de Calidad, subárea de Estadística Industrial, desarrollado en el Grupo de Investigación Gestión de la Calidad, financiado por la Universidad del Atlántico (Barranquilla, Colombia). Dirección: Carrera 30 No. 8-49, Puerto Colombia, PBX: (57) (5) 3852266. Fecha de inicio: junio de 2017. Fecha de terminación: diciembre de 2017.

2. Grupo de Investigación Gestión de la Calidad, Universidad del Atlántico (Barranquilla, Colombia). Dirección: Carrera 30 No. 8-49 Puerto Colombia, PBX: (57) (5) 3852266. ORCID ID: https://orcid.org/0000-0003-4855-4830. Correo electrónico institucional: robertoherrera@mail.uniatlantico.edu.co.

3. Grupo de Investigación Gestión de la Calidad, Universidad del Atlántico (Barranquilla, Colombia). Dirección: Carrera 30 No. 8-49 Puerto Colombia, PBX: (57) (5) 3852266. ORCID ID: https://orcid.org/0000-0001-7571-2588. Correo electrónico: paomili9@hotmail.com.

4. Grupo de Investigación Gestión de la Calidad, Universidad del Atlántico (Barranquilla, Colombia): Dirección: Carrera 30 No. 8-49 Puerto Colombia, PBX: (57) (5) 3852266 ORCID ID: https://orcid.org/0000-0001-6123-5792. Correo electrónico: karla_11231992@hotmail.com. 


\section{Introducción}

El control estadístico de procesos es esencial en el mejoramiento continuo de una organización, ya que define un procedimiento sistemático que permite realizar un seguimiento o monitoreo de una variables o variables de interés e identificary determinar las causas que generan ciertas variaciones ajenas a su desarrollo natural; además, permite definir las acciones correctivas necesarias para ajustar el proceso a las condiciones ideales o cercanas a esta (Montgomery, 2004).

En el medio, este monitoreo generalmente es aplicado asumiendo independencia entre las variables; por tanto, el seguimiento se realiza con cartas de control de Shewhart univariadas; sin embargo, es importante destacar, en los últimos años, un incremento en la metodología de control multivariada.

Entre estas técnicas se tiene la convolución entre las variables como un aporte a estas nuevas metodologías de control, dado que permite obtener una nueva variable a partir de unas ya existentes con el fin de facilitar el proceso de control de calidad. En muchos casos, es más practico realizar cartas Shewhart univariadas, ya que son las más comunes y con las que se está más familiarizado, y así evitar realizar monitoreo de control mediante cartas multivariadas. Estas cartas son las que se utilizan cuando se cuenta con más de una variable, sin saber que a partir del método de convolución se pueden reducir estas variables a una sola, y realizar monitoreo a través de cartas univariadas.

\section{Antecedentes}

En la actualidad, los consumidores de productos o servicios prestados o comercializados en las organizaciones se han convertido en clave para las organizaciones. Esto debido a la alta exigencia de los consumidores, relacionada con la globalización de todos los mercados, la cual ha hecho que las empresas se preocupen por obtener estándares de alta calidad en todos sus procesos con el propósito de alcanzar los requerimientos de los clientes y satisfacer sus necesidades mediante la comercialización de productos con un valor diferencial, y atraer y mantener a más clientes a fin de alcanzar un posicionamiento en el mercado (Pinto \& Quintero, 2017; Soto \& Rios, 2016). Para lograrlo, las organizaciones requieren mejorar sus procesos implementando mejoras mediante el control estadístico de estos que les permitan obtener una alta competitividad en el mercado.

Algunos pioneros en la utilización de técnicas estadísticas para alcanzar los requisitos del mercado mediante el control estadístico de calidad en los procesos productivos son
Taguchi (1986), Deming (1986), Ishikawa (1985), Shewhart (1931), entre otros.

En las industrias manufactureras se utilizaron estas técnicas para alcanzar altos niveles en cuanto a sus procesos, que posteriormente fueron implementadas en organizaciones con distintos procesos y en todas sus áreas (Vargas \& Cruz, 2001; Verbel, Herrera \& Maestre, 2016).

\section{Marco teórico}

Se define la convolución como un operador matemático que modifica dos funciones f1 y f2 en una tercera función, convirtiéndose en la magnitud de superposición de f1 y un tipo invertido de $\mathrm{f} 2$. La convolución vendría siendo un ejemplo de media móvil en el caso de que una de estas funciones sea función particular de un intervalo. Se llama convolución a una función que, de forma lineal y continua, transforma una señal de entrada en una nueva señal de salida (López, 2009). También se considera la convolución como una distribución de probabilidad de suma de dos variables aleatorias (Estrada, 1993). Las operaciones matemáticas involucradas en la construcción de la convolución y su implementación para alcanzar los fines deseados hacen que el proceso que se pretende realizar sea eficiente (Ballesteros, Moreno \& Gaona, 2012).

La convolución de dos funciones $\mathrm{f}_{1}(\mathrm{x})$ y $\mathrm{f}_{2}(\mathrm{x})$ definidas para todo valor de $x$ es:

$f_{1}(x) f_{2}(x)=\int_{-\alpha}^{\infty} f_{1}(x-y) f_{2}(y) d y$

La ecuación 1 se define como la integral del producto de ambas funciones después de desplazar una de ellas una a una distancia $x$, donde $f_{1}(x)$ y $f_{2}(x)$ son funciones seccionalmente continuas e integrables (Ballesteros, Moreno \& Gaona, 2012).

Un caso especial de la convolución es la suma de variables aleatorias independientes normalmente distribuida, definida de la siguiente forma,

$f_{\mathrm{Z}}(\mathrm{z})=\left[\mathrm{f}_{\mathrm{X}} * \mathrm{f}_{\mathrm{Y}}\right](\mathrm{z})=\frac{1}{2 \sqrt{\pi \sigma}} \exp \left[-\frac{1}{2 \sigma^{2}}\left(\frac{\mathrm{z}^{2}}{2}-2 \mathrm{z} \mu+2 \mu^{2}\right)\right]$

Por tanto, la suma y la diferencia de variables aleatorias independientes para la función de densidad de la distribución Normal estándar está dada por,

$$
f_{\mathrm{Z}}(\mathrm{z})=\left[\mathrm{f}_{\mathrm{X}} * \mathrm{f}_{\mathrm{Y}}\right](\mathrm{z})=\frac{1}{2 \sqrt{\pi}} \exp \left[-\frac{1}{4} \mathrm{z}^{2}\right]
$$

Por otra parte, se tienen los límites de control para convolución de: 
$L C S=D_{2} \sigma t \quad L C=T \quad L C L=D_{1} \sigma t$

Siendo T el estadístico de control de la convolución equivalente al promedio de los datos convolucionados y ot la desviación estándar de los datos convolucionados.

\section{Convolución discreta}

Su utilidad se evidencia, por ejemplo, en procesamientos digitales, donde el sistema es de tipo discreto; es el caso de las imágenes digitalizadas (Zuluaga Duque, Luque, DíazDouton, \& Pujol, 2010). La técnica de convolución es un método de solución a problemas de corrección en imágenes de tipo óptico, que consta de procedimientos matemáticos, y se constituye en un método práctico y efectivo para ser aplicado por ingenieros en una considerable cantidad de procesos (González et al., 2008), en los que se menciona la convolución utilizando únicamente su definición, debido a que solo se tienen valores en instantes discretos de tiempo. Teniendo a la mano un solo conjunto de estos valores, para convolución entre dos señales, debido a esto es estricto y necesario realizar una aproximación numérica de ella (Lagos \& Vargas, 2003). La convolución pertenece a una función de energía que permite obtener resultados significativos en el análisis de distintos procesos, incluidos los de control estadístico de calidad (Mustafa, Rodríguez \& Chauvet, 2002). El modelo matemático en forma discreta se define como:

$f(n) g(n)=\sum_{k=-\alpha}^{\alpha} f(k) g(n-k)$

donde $f, g, k$ y $n$ son la señal discreta, la ventana de convolución, la posición de la muestra, y el tamaño de dicha muestra, respectivamente (Maúlen \& Pavez, 2014).

\section{Cartas de control}

Las cartas de control son una herramienta estadística utilizada en el control estadístico de los procesos, en tanto son un instrumento útil para monitorear la estabilidad de estos, que puede detectar anormalidades en determinado momento del proceso (Merli, 2012). La calidad de los procesos se puede determinar por las fallas del producto fabricado por las compañías; si estas fallas son visibles afectan la decisión de compra por parte de los consumidores, ya que en la mayoría de los casos estos se fijan en la reputación de la compañía respecto a la calidad de sus productos. A esto se le conoce como calidad percibida, y es la razón por la cual las compañías se vieron obligadas a realizar monitoreo a sus procesos de fabricación (Montgomery, 2004). Con el objetivo de satisfacer esta necesidad de cumplir con la calidad percibida se crearon técnicas estadísticas que, aplicadas conjuntamente, componen el control estadístico de calidad; una de estas herramientas son las cartas de control (Verbel, Herrera \& Maestre, 2016).
Estas cartas fueron creadas por Shewhart (1931) y muestran en el tiempo el estado de un proceso. Estas cartas se dedican a señalar cuando existen causas especiales de variación debido a causas comunes por variaciones en el proceso, como lo son mala configuración de las máquinas, defectos de materia prima o equivocaciones de los operadores (Ballesteros, Moreno \& Gaona, 2012).

El fin de las cartas de control es analizar y controlar cómo se comporta un proceso en el tiempo. De esta manera, se distinguen variaciones asignadas a causas comunes o especiales que permiten caracterizar el funcionamiento del proceso y tomar las mejores acciones de corrección y mejoramiento. Al hablar del proceso nos referimos, generalmente, a las variables de salida; aun así, las cartas de control pueden aplicarse también para analizar la variabilidad de las variables de entrada y de control del proceso.

\section{Tipos de cartas de control}

Actualmente, hay varios tipos de cartas de control para variables o para atributos. Las de variables se utilizan para propiedades de tipo continuo, que son las mismas que necesitan un instrumento de medición (temperatura, peso, etc.). Las cartas de control más comunes son las Cartas de Shewhart. Analizando el proceso a ser controlado, se toman m muestras a intervalos regulares; las muestras correspondientes a un mismo intervalo constituyen un subgrupo. Los valores medidos se comparan con los límites obtenidos. Los límites más usados son los límites de tolerancia del proceso: límites entre los que se mueve el proceso de manera natural, se observan como límites de control (LIC, LSC) paralelos a la línea central (Merli, 2012).

Para este artículo se mencionan las cartas de control de Rango Móvil (MR), porque son las que se utilizaron. Este tipo de carta se aplica en procesos donde se selecciona una observación por subgrupo, y datos individuales, debido a condiciones técnicas económicas. El principal objetivo de este tipo de cartas es estimar la variabilidad por causas especiales cuando se presentan lecturas individuales que constituyen tendencias (Chalco-Cano, Pedraza, Román-Flores \& Álvarez-Jáuregui, 2009).

Esta investigación se desarrolla en una empresa de fabricación de resortes de compresión, donde la media 0,412 y la desviación del proceso convolucionado 0,365808 para el diámetro interno y externo son, respectivamente, $\mu=74,396$, y la desviación $\sigma=0,156$. Para el diámetro externo los parámetros son, $\mu=109,323$, y la desviación $\sigma=0,300$. Los límites estándar para Fase ll se definen como:

$\mathrm{LCS}=\mathrm{D}_{2}(2) \sigma \quad \mathrm{LC}=\mathrm{d}_{2} \sigma \quad \mathrm{LCl}: \mathrm{D}_{1}(2)$ 


\section{Estudio de caso}

El resorte de compresión es un dispositivo mecánico, de forma helicoidal y material de alambre o láminas metálicas, capaz de almacenar energía mecánica para luego liberarla y obtener como resultado una fuerza, reducir vibraciones o dar flexibilidad. Los resortes presentan diversas formas y tienen algunas características que los diferencian, como tipo de material del alambre, diámetro del alambre (d), diámetro proyectado del helicoide (D), inclinación del helicoide $(\sigma)$, cantidad de espiras (N). El resorte de compresión, denominado de esta manera porque es sometido a fuerzas de tensión con un ángulo de inclinación a que hace que sus espiras no entren en contacto al momento de la actividad, con límites en sus muelles de compresión que pueden ser: a) cerrados y cuadrados, b) cerrados y afilados, c) cerrados y abiertos, d) límites dobles, e) con o sin ganchos en una o en ambas cimas que permite fijarse a un soporte.

Estos resortes a su vez, pueden ser enrollados en dirección izquierda o derecha; permitiendo que sean de gran utilidad en la industria de automóviles, naves aéreas y bienes de consumo. En este caso de estudios se evidencia la eficiencia y robustez de las metodologías de convolución propuestas en investigaciones presentadas por (Cabrera, Hernández, Giraldo \& Dávila, 2011).

\section{Procedimiento}

Se evalúa el proceso de producción de resortes de compresión en acero. Para ello se tiene la información histórica del diámetro superior e inferior, obtenida de una empresa fabricante de estos dispositivos, las cuales se les aplica la convolución ecuación (1), adicionado para ello, el empleo de álgebra convolutiva (Muñoz, 2007); obteniendo una nueva variable en el proceso de fabricación de los resortes, a la cual se le hace monitoreo en línea utilizando las cartas de control de Rango Móvil, que permitirán analizar las etapas del proceso y evaluar su impacto de influencia en el producto final conformado por estas dos variables $X$ y $Y$, que se acoplan dada una tolerancia entre estas, se asume un comportamiento normal para cada uno de los elementos del proceso, identificando sus parámetros tanto la medida de localización como la medida de variabilidad, para las mediciones respectivas que se hicieron a cada elemento.

En cada subgrupo se determina el estadístico de control para cada subgrupo, la nueva variable aleatoria convolucionada basados en los parámetros definidos con anterioridad; obteniendo una nueva variable proveniente de la convolución de las variables originales. Esta nueva variable, ecuación (1), se define como $Z=X-Y \circ Z=X+Y$.
Esta resta aleatoria llamada Z es el estadístico de control para las cartas convolucionadas. Se hallaron los límites de control de rango móvil (ecuación (3)). Se elaboró la carta de control para variables obtenidas mediante convolución de rango móvil para Fase II. Luego se encontraron los parámetros $\mu$ y $\sigma$ del proceso convolucionado, y se elaboró una carta de rango móvil para las variables históricas de los diámetros interno y externo del resorte; por último, se compararon los resultados obtenidos de las cartas para las variables individuales y para las variables convolucionadas.

\section{Resultados}

La Tabla 1 presenta las mediciones realizadas en la fabricación de resortes de compresión, donde se miden dos características de calidad de interés: el diámetro interno y el diámetro externo.

Un resorte de compresión es un mecanismo con forma de hélice, generalmente fabricado en alambre, diseñado para resistir las fuerzas de compresión aplicadas por la energía del movimiento. Los resortes de compresión se pueden localizar en muchas aplicaciones, desde el sector aeroespacial hasta en bienes de consumo. El más comercializado es el resorte cilíndrico; utilizado en asentamiento de energía, así como en la deflexión de carga. Los muelles helicoidales cilíndricos son los que presentan mejores atributos debido a que soportan tensiones inferiores a los otros tipos.
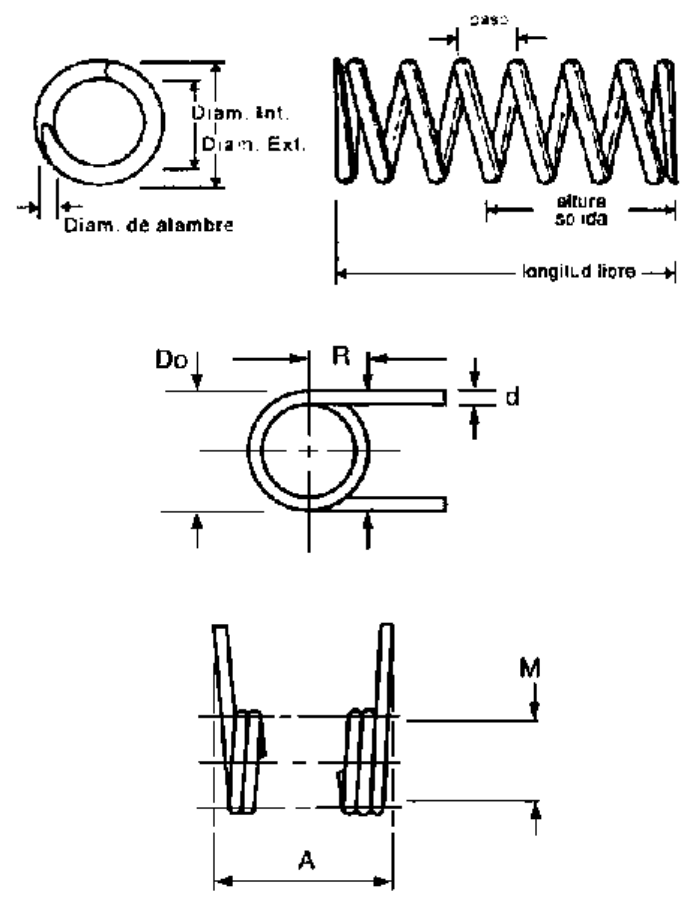

Figura 1. Esquema de un resorte de comprensión, acero 302 inoxidable, alambre acerado. Fuente: Autores. 
Tabla 1

Diámetros del resorte y variables convolucionadas, acero 302 inoxidable, alambre acerado

\begin{tabular}{ccc}
\hline Diámetro interno & Diámetro externo & Variables convolucionadas \\
\hline 74,50 & 109,65 & 35,15 \\
74,76 & 109,80 & 35,04 \\
74,50 & 108,60 & 34,10 \\
74,40 & 109,35 & 34,95 \\
74,40 & 109,10 & 34,70 \\
74,12 & 109,43 & 35,31 \\
74,50 & 109,55 & 35,05 \\
74,50 & 109,05 & 34,55 \\
74,50 & 109,50 & 35,00 \\
74,50 & 109,50 & 35,00 \\
74,50 & 109,50 & 35,00 \\
74,50 & 109,50 & 35,00 \\
74,18 & 109,47 & 35,29 \\
74,40 & 109,22 & 34,82 \\
74,08 & 109,44 & 35,36 \\
74,08 & 109,13 & 35,05 \\
74,40 & 108,83 & 34,43 \\
74,70 & 109,05 & 34,35 \\
74,30 & 109,73 & 35,43 \\
74,10 & 109,05 & 34,95 \\
\hline
\end{tabular}

Fuente: Autores.

Son conocidos los parámetros del proceso: un promedio de rango móvil para variables convolucionadas de 0,412. Una desviación estándar sigma $\sigma$ del proceso, estimado a partir del promedio de rango móvil de 0,365. Los límites de control de rango móvil para variables convolucionadas obtenidos son: $\mathrm{LSC}=1,348$ límite superior, $\mathrm{LC}=0,412$, límite central y el límite inferior es $\mathrm{LCl}=0,000$, (ecuación (2)), (ver Figura 2).

Por otra parte, se obtuvieron los límites para datos individuales de los diámetros del resorte (ecuación (4)), estos son: para el diámetro interno $L S C=0,722, L C=0,221$ y el límite inferior es $\mathrm{LCl}=0,000$ y para el diámetro externo: el límite superior $\mathrm{LSC}=$ 1,138 , el límite central $L C=0,348$ y el límite inferior es $L C l=0,000$; se elaboró una carta para valores individuales de los diámetros interno y externo para determinar si los datos se encuentran en un estado de control estadístico (ver figuras 3 y 4).

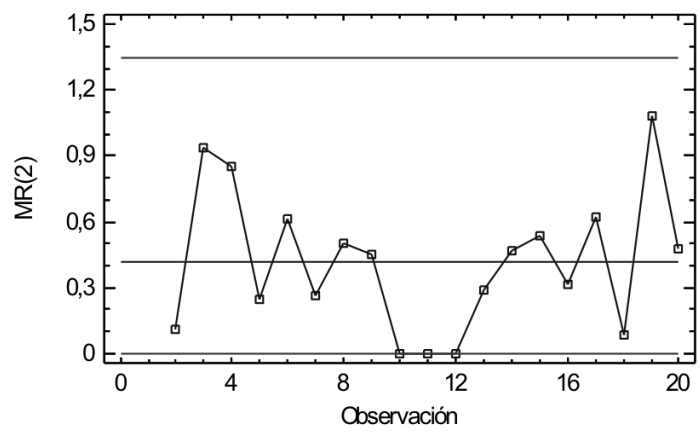

Figura 2. Carta de control convolución rango móvil, acero 302 inoxidable, proveniente de la Tabla 1. Fuente: Autores.

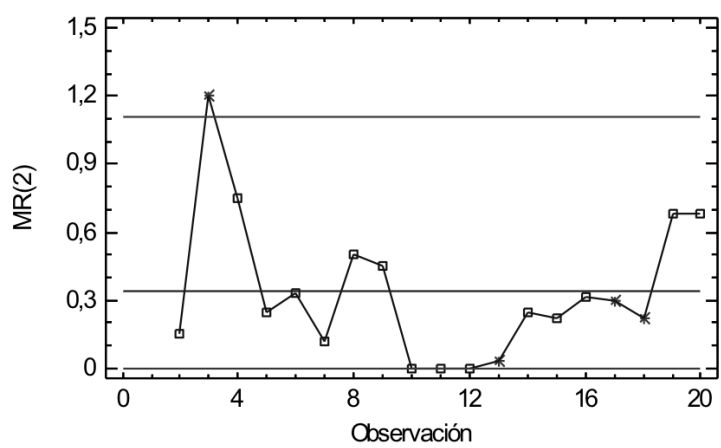

Figura 3. Carta de control rango móvil para diámetro externo del acero 302 Inoxidable. Fuente: Autores.

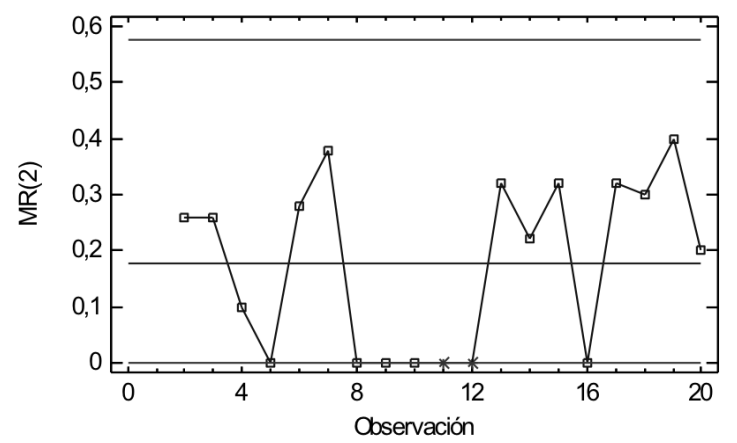

Figura 4. Carta de control rango móvil para diámetro interno, acero 302 inoxidable. Fuente: Autores.

Se puede apreciar en la Figura 1, después de haber realizado la carta de control de rango móvil en Fase II, que los subgrupos están dentro de los límites de control; de igual manera, se observa que hubo tendencia entre los puntos, lo que ocasiona que estos tengan tendencias descendentes y ascendentes alrededor del límite central.

Se aprecia en la Figura 3 que hay un punto fuera de los límites de control, es decir un estado fuera de control, mientras que en la Figura 4 los subgrupos están dentro de los límites de control. Esto nos lleva a concluir que al realizar la convolución de las variables no se pueden determinar los estados fuera de control detectados en las cartas individuales de los rangos móviles.

\section{Conclusiones}

Las cartas de control, sin lugar a dudas, son una de las herramientas tradicionales del control estadístico de proceso CEP; permiten el monitoreo de variables o características de calidad de un producto o proceso, y son de gran utilidad para identificar señales de alarma o puntos con un comportamiento no aleatorio.

La convolución, como se observa en el caso de estudio, influye en la interpretación de los resultados debido a 
que la nueva variable convolucionada puede minimizar el impacto de una de las variables afectadas por un factor ajeno al proceso; es decir, si existe una señal de alarma por una causa asignable en una de las variables, tal como se presentó en el diámetro externo (Figura 3), la convolución permite encubrir este incremento de la variabilidad, evaluando en forma global el comportamiento del proceso; obteniendo resultados opuestos, si el monitoreo se realiza en forma independiente en cada variable.

La razón principal de realizar la convolución es crear cartas univariadas, evitando de esta manera ecuaciones más complejas presentadas en el campo multivariado en los casos que se tenga más de una variable; con la ventaja adicional de reducir el tiempo y los recursos de la organización (Maúlen \& Pavez, 2014).

Es importante resaltar que en muchos de los procesos productivos es difícil controlar algunas de las variables por su alta variabilidad, es el caso de productos a granel. En este caso, realizar un control univariado tradicional para cada una de las variables que caracterizan el producto implica inhibirse de presentar un análisis global del proceso. Este inconveniente es superado cuando se seleccionan variables mixtas, propósito de la convolución en el monitoreo del estadístico de control en su medida de variabilidad, transformadas en una nueva variable, posteriormente presentada como un caso estrictamente univariado.

\section{Agradecimientos}

Resaltamos la colaboración brindada por la Universidad del Atlántico en la realización del presente trabajo. Agradecemos especialmente al Grupo de Investigación Gestión de la Calidad con su semillero de investigación Estadística Industrial.

\section{Referencias}

Ballesteros, D., Moreno, M. \& Gaona, A. (2012). Comprensión de señales ECG sobre FPGA utilizando un esquema modificado de convolución de la transformada Wavelet Discreta. Ingeniare. Revista Chilena de Ingeniería, 20(1), 8-16.

Cabrera, A. M., Hernández, J. G., Giraldo, O. D. \& Dávila, L. F. (2011). Simplificación de señales Senoidales y Cosenoidales mediante triángulos de cano obtenidos usando propiedades de la convolución. Scientia et Technica, 2(48), 233-238.

Chalco-Cano, Y., Pedraza Arpasi, J., Román-Flores, H. \& Álvarez-Jáuregui, G. (2009). Una aproximación de números difusos de tipo trapezoidal. Ingeniare. Revista Chilena de Ingeniería, 17(2), 158-165.
Deming, W. E. (1986). Out of the crisis. Massachusetts Institute of Technology. Cambridge: Center for advanced engineering study.

Estrada, H. (1993). Evaluación de integrales de dos electrones mediante el teorema de convolución de Fourier. MOMENTO, (9), 36-40.

González, M., Rodríguez, F. J., Maciel, J. M., Romo, J. C., Rosas, F. J., De Luna Ortega, C. A., ... Lecona, F. G. (2008). Reducción de ruido digital en señales ECG utilizando filtraje por convolución. Investigación y Ciencia, 16(40), 26-32

Ishikawa, D. K. (1985). What Is Total Quality Control?: The Japanese Way (Business Management). Prentice Hall Trade.

Lagos, I. \& Vargas, J. (2003). Sistema de familias de distribuciones de Johnson, una alternativa para el manejo de datos no normales en cartas de control. Revista Colombiana de Estadística, 26(1), 25-40.

López, H. I. (2009). Método alternativo para calcular la convolución de señales en tiempo continuo. En Ponencia Realizada en el Primer Congreso Regional de Electricidad, Electrónica y Sistemas, CREES.

Maúlen, D. \& Pavez, J. (2014). Control Estadístico de Procesos, Implementación de cartas de control en paquete estadístico $R$.: Universidad de Valparaíso.

Merli, G. (2012). Gestión de la calidad: Control estadístico y Seis Sigma. Telos, 14(22), 269-286.

Montgomery, D. (2004). Control Estadístico de la Calidad (3a. ed.): Limusa Wiley.

Muñoz, D. (2007). Corrección de la distorsión de imágenes en equipos ópticos por medio de splines y técnicas de análisis de imágenes. Scientia et Technica, 13(35), 383-388.

Mustafa, A. M., Rodríguez, N. L. \& Chauvet, S. (2002). Control de calidad: Cartas de control por variables. En Congreso Regional de Ciencia y Tecnología NOA 2002. Argentina: Secretaría de Ciencia y Tecnología, Universidad Nacional de Catamarca.

Pinto, T. Y. \& Quintero, H. C. (2017). Modelo de negocios para comercialización de películas y recubrimientos comestibles en Bucaramanga. I+D Revista de Investigaciones, 10(2), 14-24.

Shewhart, W. (1931). The Economic Control of Quality of Manufactured Products. New York: David Van Nostrand.

Soto, I. O. \& Rios, C. P. (2016). Comportamiento y experiencia de consumo desde la interconexión e interactividad de la World Wide Web: un recorrido teórico. I+D Revista De Investigaciones, 8(2), 35-45.

Taguchi, G. (1986). Introduction to Quality Engineering: Designing Quality Into Products and Processes. Tokyo: Asian Productivity Organization.

Vargas, M. G. \& Cruz, E. A. (2001). Estudio del Efecto de las Máscaras de Convolución en Imágenes Mediante 
el Uso de la Transformada de Fourier. Ingeniería e Investigación, (48), 46-51.

Verbel, A., Herrera, R. J. \& Maestre, K. (2016). Aplicación de nuevas propuestas multivariantes para medir la capacidad de un proceso. Ingeniare, 12(21), 31-42.

Zuluaga, Duque, H., Luque, S. O., Díaz-Douton, F., \& Pujol, J. (2010). Medida de la calidad óptica de lentes intraoculares multifocales in vitro mediante un sistema de paso simple y doble paso modificado. Barcelona: Terassa. 